\title{
Genetic Relationship among Sweetpea Cultivars and Related Species by AFLP
} Analysis

\author{
Kaoru Nakamura ${ }^{1,2 *}$, Toru Sugita ${ }^{1}$, Hidenori Tanaka ${ }^{3}$ and Ryo Akashi ${ }^{3}$ \\ ${ }^{1}$ Miyazaki Agricultural Research Institute, Miyazaki 880-0212, Japan \\ ${ }^{2}$ Interdisciplinary Graduate School of Agriculture and Engineering, University of Miyazaki, Miyazaki 889-2192, Japan \\ ${ }^{3}$ Frontier Science Research Center, University of Miyazaki, Miyazaki 889-2192, Japan
}

\begin{abstract}
Sweetpea (Lathyrus odoratus L.) is an important ornamental crop belonging to the Fabaceae family. In Japan, commercial cut flower production under forced culture has flourished, but few cultivars are bred exclusively for cut flower forced culture. In order to obtain knowledge for the selection of breeding materials, we elucidated the genetic relationships among eighty-seven sweetpea cultivars and two related species using the amplified fragment length polymorphisms (AFLP). Sweetpea cultivars were grouped into two clusters. Cluster I consisted of four cultivars, including a wild cultivar, 'Cupani' and cultivars established from spontaneous mutation or natural hybridization, such as 'Cupid' and 'Painted Lady'. The biggest cluster, cluster II, consisted of eighty-three sweetpea cultivars. No distinct subcluster shared common morphological or ecological characteristics, such as tendril-less, plant type, or flowering habit in cluster II. The distinct genetic differentiation among cultivars highly seems to reflect sweetpea breeding history originating in the United Kingdom 300 years ago using limited genetic resources. Thus, hybridization with genetically distinct, exotic resources may contribute to the development of sweetpea cultivars.
\end{abstract}

Key Words: AFLP, cluster analysis, cultivar, relationship, sweetpea.

\section{Introduction}

Genetic resources used as breeding materials involve cultivars or lines bred by breeders, native species adapted to the natural conditions of specific areas, and wild species. These genetic resources have genetic variability or latent potentialities to spread variation (Ohkawa, 1995). It is very important to collect genetic resources, and maintain, reproduce, and examine their morphologies, reveal relationships, and to identify and classify such resources to be used in future plant breeding. Recently, DNA-based markers for genetic analysis have been used to examine relationships, and to identify and classify cultivars and lines (Tsuruta et al., 2002). Notably, amplified fragment length polymorphisms (AFLP) analysis is an efficient technique to reveal genetic relationships, and to identify and classify cultivars and lines. Hence, the AFLP technique is used already in many crops to determine genetic relationships (Lee and Han, 2006; Mace et al., 1999; Warwick et al., 2007).

Received; December 2, 2009. Accepted; March 31, 2010.

* Corresponding author (E-mail: nakamura-kaoru@pref.miyazaki.lg.jp).
Sweetpea is an important ornamental crop. In Japan, commercial cut flower production under forced culture has flourished (Motozu, 2006; Nakamura et al., 2008). Because sweetpea was improved in the United Kingdom and the United States and then introduced into Japan (Inoue, 2007; Nakamura et al., 2008), most cultivars used for cut flower production in Japan were selected and fixed from imported winter or spring flowering cultivars from the United States (Inoue, 2007; Nakamura et al., 2008). Therefore, few cultivars have been bred by hybridization specific for cut flower forced culture in Japan. Commercial cultivars for cut flowers need good characteristics, including not only flower color, flower size, flower form, long vase life, and tolerance to bud drop, but also high yielding ability, disease resistance, plant vigor stability, and heat tolerance. Hereafter, it is important to reveal the genetic relationships and examine the specific characteristics of each distinct genetic group in order to choose cultivars adapted to breeding objectives.

The sweetpea has a history of more than 300 years. Its characteristics, such as flower color, flower form, and flowering habit have diversified significantly as the result 
of breeding throughout its history. The morphological and ecological characteristics of sweetpea cultivars under normal culture were described previously by Beal (1914), Ito et al. (1991), and Hanada (2003). Murray et al. (1992) reported no evidence of change in chromosome structure and genome size throughout sweetpea development. On the other hand, Hanada and Hirai (2000) reported that the RAPD technique classified 15 cultivars into clusters corresponded to the flowering habit and the tendril-less trait. However, it is unknown whether there is a relationship between these characteristics and the genetic differentiation of other sweetpea cultivars.

We have been breeding sweetpea cultivars for commercial cut flower production under forced culture (Nakamura et al., 2008, 2006a, b). We have also been collecting cultivars used for not only cut flower production in Japan but also those used as summer flowering cultivars from the United Kingdom to obtain useful characteristics, and reported the morphological and ecological classification of cultivars under forced culture (Nakamura et al., 2010).

In this study, in order to obtain knowledge for the selection of breeding materials, we elucidated the genetic relationships among eighty-seven sweetpea cultivars and two related species using the AFLP technique.

\section{Materials and Methods}

\section{Plant materials and DNA extraction}

One accession each of 87 sweetpea cultivars and two out-group species, L. belinensis Maxted \& Gloyder and L. chloranthus Boiss. were used in this study (Table 1). The plant materials were grown in the greenhouse of the Miyazaki Agricultural Research Institute. Young leaves $(0.1 \mathrm{~g})$ of each cultivar and species were frozen in liquid nitrogen and ground into a fine powder using a mortar and pestle. DNA was extracted from powder using the CTAB method, as described by Murray and Thompson (1980), and Nucleon PHYTOPURE (Amersham Life Science, Buckinghamshire, UK).

\section{AFLP analysis}

The amplification conditions adopted for AFLP analysis were initially described by Vos et al. (1995). The electrophoresis conditions and band detection procedures were adopted for high efficiency genome scanning (HEGS) system as described by Kawasaki and Murakami (2000) and Kawasaki et al. (2003). Genomic DNA (250 ng) was digested with Mse I (5 units) and $X b a$ I (5 units). Digested fragments were ligated to each adaptor (Table 2) of the restriction enzyme combination. The first amplification was performed using preamplification primers (Table 2). The first PCR products were diluted $1 / 100$ with $1 / 10$ TE $(1 \mathrm{mM}$ Tris hydroxymethyl aminomethane, $0.1 \mathrm{mM}$ disodium dihydrogen ethylendiamine tetraacetate dehydrate, $\mathrm{pH} 8.0$ ) buffer and used as the template DNA for the second amplification step. The second amplification was performed using primers (Table 2) in a $10 \mu \mathrm{L}$ total reaction volume. Primer combinations for amplification were: Mse I-caa/Xba I-cgc, Mse I-cac/Xba I-ccg, Mse $\mathrm{I}-\mathrm{cac} / X b a$ I-cgc, Mse I-cag/Xba I-cat, Mse I-cag/Xba I-cga, Mse I-cag/Xba I-cgt, Mse I-cat/Xba I-cgc, Mse I-cat/Xba I-cgg, Mse I-cat/Xba I-cgt, $M s e \mathrm{I}$-cat/Xba I-cta, $M s e$ I-cca/Xba I-cac, Mse I-cca/Xba I-cag, Mse I-cca/ $X b a \mathrm{I}-\mathrm{ccg}$, Mse I-cca/Xba I-cgc, Mse I-ccc/Xba I-ccc, $M s e$ I-ccc/Xba I-cgc, Mse I-ccc/Xba I-cgg, Mse I-ccc/ $X b a$ I-cgt, Mse I-ccc/Xba I-ctc, Mse I-ccc/Xba I-ctg, Mse I-ccc/Xba I-ctt. The PCR products of the second amplification were separated on $13 \%$ polyacrylamide gels at $300 \mathrm{~V}$ for $10 \mathrm{~min}$ and $450 \mathrm{~V}$ for $3 \mathrm{~h}$ in a small vertical electrophoresis unit of $26 \times 26 \mathrm{~cm}$ (Nippon-eido, Tokyo, Japan). The gels were stained with SYBR Green I (BMA, Rockland, USA) for $30 \mathrm{~min}$, and were detected with a fluorescent image analyzer (FLA-3000G; Fuji Film, Tokyo, Japan). Duplicate amplifications were analyzed to confirm the AFLP analyses.

\section{Data analysis}

Polymorphic AFLP markers were manually scored as binary data with presence of a band denoted as " 1 " and absence as " 0 ". Only clearly distinguishable bands were used in the genetic analysis. Genetic distances were estimated using the simple matching coefficient (Sokal and Michener, 1958). Similarity coefficient was calculated as $\mathrm{S} i j=(a+d) /(a+b+c+d)$ : where $\mathrm{S} i j$ is the similarity between two individuals $i$ and $j, a$ is the number of fragments in both $i$ and $j, b$ is the number of fragments present in $i$ and absent in $j, c$ is the number of fragments absent in $i$ and present in $j$, and $d$ is the number of fragments absent in both $i$ and $j$. Conversion to genetic distances, D $i j$, was obtained by the equation $\mathrm{D} i j=1-$ $\mathrm{S} i j$. The genetic distances obtained were used for cluster analysis based on the neighbor-joining (NJ) method (Saitou and Nei, 1987) with 1000 bootstraps by using the software package TREECON (ver. 1.3b) (Van De Peer and De Wachter, 1994).

\section{Results and Discussion}

\section{AFLP polymorphism}

The 21 AFLP primer combinations used in this study amplified 1043 DNA fragments among eighty-seven sweetpea cultivars and two related species accessions (Table 3). The number of DNA fragments for each primer combination ranged from 30 to 71 (mean, 49.7). Out of 1043 amplified bands, 159 (15.2\%) were found to be polymorphic among accessions, and each primer combination yielded 4 to 16 polymorphic bands. The primer combination of Mse I-cat/Xba I-cgg generated the most polymorphic bands; therefore, we suggest that AFLP analysis is a useful genetic technique to study relationships in sweetpea cultivars. 
Table 1. Characteristics and origins of sweetpea cultivars and related two Lathyrus species used in this study.

\begin{tabular}{|c|c|c|c|c|c|c|c|}
\hline \multirow{2}{*}{ Species and cultivar } & \multirow{2}{*}{$\begin{array}{l}\text { Usage in } \\
\text { Japan }\end{array}$} & \multicolumn{2}{|c|}{ Color of the standard ${ }^{2}$} & \multirow{2}{*}{$\begin{array}{c}\text { Flowering } \\
\text { habit }^{y}\end{array}$} & \multirow[b]{2}{*}{ Tendril $^{\mathrm{x}}$} & \multirow[b]{2}{*}{ Plant type ${ }^{y}$} & \multirow[b]{2}{*}{ Origin } \\
\hline & & $\begin{array}{c}\text { Color } \\
\text { code No. }\end{array}$ & Color name & & & & \\
\hline \multicolumn{8}{|l|}{ Lathyrus odoratus $\mathrm{L}$. } \\
\hline Aisome & Cut flower & 8905 & bright reddish purple & Spring & + & Tall & Bred in Miyazaki Agri. Res. Inst. \\
\hline Anniversary & Garden & 9501 & pale purplish pink & Summer & + & Tall & Selfed and selected after introduced from seed company \\
\hline Ayahime & Cut flower & 1615 & pale grayish brown & Spring & + & Tall & Bred in Miyazaki Agri. Res. Inst. \\
\hline Benishikibu & Cut flower & 0110 & dark red & Spring & + & Tall & Bred in Miyazaki Agri. Res. Inst. \\
\hline Blue Ripple & Garden & 8615 & light grayish purple & Summer & + & Tall & Mis. Noguchi \\
\hline Celeste & Cut flower & 8304 & light bluish purple & Spring & + & Tall & Selfed and selected after introduced from seed company \\
\hline Ceremony Lavender & Cut flower & 8604 & bright purple & Winter & - & Tall & Selfed and selected after introduced from seed company \\
\hline Ceremony Pink & Cut flower & 9704 & strong purplish pink & Winter & - & Tall & Selfed and selected after introduced from seed company \\
\hline Ceremony Scarlet & Cut flower & 0714 & deep yellowish red & Winter & - & Tall & Selfed and selected after introduced from seed company \\
\hline Ceremony White & Cut flower & 2501 & yellowish white & Winter & - & Tall & Selfed and selected after introduced from seed company \\
\hline Charlie's Angel & Garden & 8304 & light bluish purple & Summer & + & Tall & Selfed and selected after introduced from seed company \\
\hline Christina & Cut flower & 2901 & yellowish white & Winter & + & Tall & Selfed and selected after introduced from seed company \\
\hline Cupani & Wild type & 0419 & grayish red & Summer & + & Tall & Mis. Kurose \\
\hline Cupid & Bedding & 9505 & strong purplish pink & Spring & + & Dwarf & Selfed and selected after introduced from seed company \\
\hline Diana & Cut flower & 0105 & strong pink & Winter & + & Tall & Daiichi Engei co.ltd. \\
\hline Early Lavender & Cut flower & 8604 & bright purple & Winter & + & Tall & Selfed and selected after introduced from seed company \\
\hline Early Salmon Pink & Cut flower & 0111 & pink & Spring & + & Tall & Selfed and selected after introduced from seed company \\
\hline Early White & Cut flower & 3301 & yellowish white & Winter & + & Tall & Selfed and selected after introduced from seed company \\
\hline Easter Parade & Cut flower & 3301 & yellowish white & Spring & + & Tall & Selfed and selected after introduced from seed company \\
\hline Elizabeth & Cut flower & 9704 & strong purplish pink & Winter & + & Tall & Selfed and selected after introduced from seed company \\
\hline Ellie & Cut flower & 8604 & bright pink & Winter & + & Tall & Selfed and selected after introduced from seed company \\
\hline Emily & Cut flower & 0404 & strong pink & Winter & + & Tall & Selfed and selected after introduced from seed company \\
\hline First Lady & Cut flower & 9503 & purplish pink & Spring & + & Tall & Hamayu agricultural cooperatives \\
\hline Galatia & Cut flower & 8310 & strong bluish purple & Winter & + & Tall & Selfed and selected after introduced from seed company \\
\hline Grace & Cut flower & 8604 & bright purple & Spring & + & Tall & Osuzu agricultural cooperatives \\
\hline Honeymoon & Garden & 8303 & light bluish purple & Summer & + & Tall & Selfed and selected after introduced from seed company \\
\hline Maifuji & Cut flower & 8604 & bright purple & Spring & + & Tall & Bred in Miyazaki Agri. Res. Inst. \\
\hline Maihime & Cut flower & 0114 & strong red & Spring & + & Tall & Bred in Miyazaki Agri. Res. Inst. \\
\hline Mammoth Deep Rose & Cut flower & 9707 & vivid purplish red & Winter & + & Tall & Selfed and selected after introduced from seed company \\
\hline Mary & Cut flower & 0104 & strong pink & Winter & + & Tall & Selfed and selected after introduced from seed company \\
\hline Mimi & Cut flower & 0104 & strong pink & Spring & + & Tall & Bred in Miyazaki Agri. Res. Inst. \\
\hline Miranda & Cut flower & 0105 & strong pink & Winter & + & Tall & Selfed and selected after introduced from seed company \\
\hline Miyazaki Sunny Peach & Cut flower & 0702 & pale yellowish pink & Spring & + & Tall & Bred in Miyazaki Agri. Res. Inst. \\
\hline Mrs. Douglas MacArthur & Cut flower & 0105 & strong pink & Winter & + & Tall & Selfed and selected after introduced from seed company \\
\hline Musica Purple & Cut flower & 9208 & vivid red purple & Winter & - & Tall & Bred in Miyazaki Agri. Res. Inst. \\
\hline Musica Rose & Cut flower & 9205 & strong purplish pink & Spring & - & Tall & Bred in Miyazaki Agri. Res. Inst. \\
\hline Neo Grace & Cut flower & 8905 & bright reddish purple & Spring & + & Tall & Hamayu agricultural cooperatives \\
\hline Osuzu Royal White & Cut flower & 3301 & yellowish white & Spring & + & Tall & Osuzu agricultural cooperatives \\
\hline Painted Lady & Wild type & 0106 & bright red & Summer & + & Tall & Dr. Inoue \\
\hline Pansy Lavender Flash & Garden & 8604 & bright purple & Summer & + & Dwarf & Tompson \& Morgan Seed \\
\hline Patio Deep Purple & Bedding & 9218 & dark grayish purple & Spring & + & Dwarf & Selfed and selected after introduced from seed company \\
\hline Patio Pink & Bedding & 9705 & strong purplish pink & Spring & + & Dwarf & Selfed and selected after introduced from seed company \\
\hline Patio Rose & Bedding & 9708 & strong purplish pink & Spring & + & Dwarf & Selfed and selected after introduced from seed company \\
\hline Patio Rose Pink & Bedding & 9705 & strong purplish pink & Spring & + & Dwarf & Selfed and selected after introduced from seed company \\
\hline Pearl & Cut flower & 9202 & pale orchid & Spring & + & Tall & Hamayu agricultural cooperatives \\
\hline Percy Thrower & Garden & 9205 & strong purplish pink & Summer & + & Tall & Selfed and selected after introduced from seed company \\
\hline Red Arrow & Garden & 0713 & strong yellowish red & Summer & + & Tall & Selfed and selected after introduced from seed company \\
\hline Rosé & Cut flower & 9205 & strong purplish pink & Spring & + & Tall & Osuzu agricultural cooperatives \\
\hline Rose Pink & Cut flower & 9205 & strong purplish pink & Spring & + & Tall & Selfed and selected after introduced from seed company \\
\hline Royal blue & Cut flower & 8310 & strong bruish purple & Spring & + & Tall & Selfed and selected after introduced from seed company \\
\hline Royal Lavender & Cut flower & 8304 & light bluish purple & Spring & + & Tall & Selfed and selected after introduced from seed company \\
\hline Royal Marion & Cut flower & 2503 & pale yellow & Spring & + & Tall & Selfed and selected after introduced from seed company \\
\hline Royal Navy blue & Cut flower & 8609 & dark purple & Spring & + & Tall & Selfed and selected after introduced from seed company \\
\hline Royal Rose Pink & Cut flower & 9204 & strong purplish pink & Spring & + & Tall & Selfed and selected after introduced from seed company \\
\hline Royal Wedding & Cut flower & 2903 & pale greenish purple & Spring & + & Tall & Selfed and selected after introduced from seed company \\
\hline Royal White & Cut flower & 3302 & yellowish white & Spring & + & Tall & Selfed and selected after introduced from seed company \\
\hline Sakuragawa & Cut flower & 8902 & pale purplish pink & Spring & + & Tall & Bred in Miyazaki Agri. Res. Inst. \\
\hline Shikibu & Cut flower & 0110 & dark red & Spring & + & Tall & Bred in Miyazaki Agri. Res. Inst. \\
\hline Silky Cherry & Cut flower & 9203 & purplish pink & Spring & + & Tall & Bred in Miyazaki Agri. Res. Inst. \\
\hline Silky Peach & Cut flower & 1315 & pale brown & Winter & + & Tall & Bred in Miyazaki Agri. Res. Inst. \\
\hline Spring Fair & Cut flower & 0104 & strong pink & Spring & + & Tall & Takachiho agricultural cooperatives \\
\hline Stella & Cut flower & 2503 & pale yellow & Spring & + & Tall & Selfed and selected after introduced from seed company \\
\hline
\end{tabular}


Table 1. Continued.

\begin{tabular}{|c|c|c|c|c|c|c|c|}
\hline \multirow[b]{2}{*}{ Species and cultivar } & \multirow{2}{*}{$\begin{array}{l}\text { Usage in } \\
\text { Japan }\end{array}$} & \multicolumn{2}{|c|}{ Color of the standard ${ }^{2}$} & \multirow{2}{*}{$\begin{array}{c}\text { Flowering } \\
\text { habit }^{y}\end{array}$} & \multirow[b]{2}{*}{ Tendril $^{x}$} & \multirow[b]{2}{*}{ Plant type ${ }^{y}$} & \multirow[b]{2}{*}{ Origin } \\
\hline & & $\begin{array}{l}\text { Color } \\
\text { code No. }\end{array}$ & Color name & & & & \\
\hline Super Lavender & Cut flower & 8604 & bright purple & Spring & + & Tall & Selfed and selected after introduced from seed company \\
\hline Super Mid Blue & Cut flower & 8310 & strong bluish purple & Spring & + & Tall & Selfed and selected after introduced from seed company \\
\hline Super Rose & Cut flower & 9205 & strong purplish pink & Spring & + & Tall & Selfed and selected after introduced from seed company \\
\hline Super Scarlet & Cut flower & 0406 & bright red & Spring & + & Tall & Selfed and selected after introduced from seed company \\
\hline Super Snoopea Pale Purple & Garden & 8310 & strong bluish purple & Summer & - & Tall & A mutant of 'Super Snoopea Purple' \\
\hline Super Snoopea Pink & Garden & 9205 & strong purplish pink & Spring & - & Medium & Selfed and selected after introduced from seed company \\
\hline Super Snoopea Purple & Garden & 9218 & dark grayish purple & Spring & - & Medium & Selfed and selected after introduced from seed company \\
\hline Super Snoopea Red & Garden & 0409 & deep red & Spring & - & Medium & Selfed and selected after introduced from seed company \\
\hline Super White & Cut flower & 3302 & yellowish white & Spring & + & Tall & Selfed and selected after introduced from seed company \\
\hline Susie & Cut flower & 0104 & strong pink & Winter & + & Tall & Selfed and selected after introduced from seed company \\
\hline Sweet Memory Blue & Garden & 8310 & strong bluish purple & Summer & + & Tall & Selfed and selected after introduced from seed company \\
\hline Sweet Memory Purple & Garden & 0410 & dark red & Summer & + & Tall & Selfed and selected after introduced from seed company \\
\hline Sweet Memory Rose & Garden & 0106 & bright red & Summer & + & Tall & Selfed and selected after introduced from seed company \\
\hline Tendril-less Honeymoon & Garden & 8602 & pale purple & Summer & - & Tall & A mutant of 'Honeymoon' \\
\hline Utahime & Cut flower & 0415 & strong red & Winter & + & Tall & Bred in Miyazaki Agri. Res. Inst. \\
\hline View Pink & Bedding & 9704 & strong purplish pink & Winter & + & Dwarf & Selfed and selected after introduced from seed company \\
\hline View Purple & Bedding & 8613 & dull purple & Winter & + & Dwarf & Selfed and selected after introduced from seed company \\
\hline View White & Bedding & 3301 & yellowish white & Winter & + & Dwarf & Selfed and selected after introduced from seed company \\
\hline White Giant & Cut flower & 3301 & yellowish white & Winter & + & Tall & Selfed and selected after introduced from seed company \\
\hline Winter Elegance Deep Rose & Cut flower & 9707 & vivid purplish red & Winter & + & Tall & Selfed and selected after introduced from seed company \\
\hline Winter Elegance Lavender & Cut flower & 8604 & bright purple & Winter & + & Tall & Selfed and selected after introduced from seed company \\
\hline Winter Elegance Pink Diana & Cut flower & 9504 & strong purplish pink & Winter & + & Tall & Selfed and selected after introduced from seed company \\
\hline Winter Elegance Salmon & Cut flower & 0105 & strong pink & Winter & + & Tall & Selfed and selected after introduced from seed company \\
\hline Winter Elegance Scarlet & Cut flower & 0707 & deep reddish brown & Winter & + & Tall & Selfed and selected after introduced from seed company \\
\hline Winter Flowering Lavender & Cut flower & 8604 & bright purple & Winter & + & Tall & Selfed and selected after introduced from seed company \\
\hline L. belinensis Maxted \& Gloyder. & Garden & 1308 & deep brown & Summer & + & Tall & Tompson \& Morgan Seed \\
\hline L. chloranthus Boiss. & Garden & 2911 & strong greenish yellow & Summer & + & Medium & Dr.Inoue \\
\hline
\end{tabular}

z Japan horticultural plant standard (JHS) color chart. 1984. (Japan. Color Res. Inst., Tokyo, Japan)

${ }^{y}$ Ito et al. (1991)

$x+$ : presence of tendril, - : absence of tendril

Table 2. Nucleotide sequences of the primers and adapters used in AFLP analysis.

\begin{tabular}{lll}
\hline \hline \multicolumn{2}{c}{ Primers/Adapters } & \multicolumn{1}{c}{ Nucleotide sequences } \\
\hline Mse I & adapter 1 & 5'-gac gat gag tcc tga g-3' \\
Mse I & adapter 2 & 5'-tac tca gga ctc at-3' \\
Xba I & adapter 1 & 5'-ctc gta gac tgc gta cc-3' \\
Xba I & adapter 2 & 5'-cta ggg tac gca gtc tac-3' \\
Mse I & primer (+1) & 5'-gat gag tcc tga gta ac-3' \\
Xba I & primer (+1) & 5'-gac tgc gta ccc tag ac-3' \\
Mse I & primer (+3) & 5'-gat gag tcc tga gta ac+aa(M1), ac(M2), \\
& & ag(M3), at(M4), ca(M5), cc(M6)-3' \\
Xba I & primer (+3) & 5'-gac tgc gta ccc tag ac+ac(X2), ag(X3), \\
& & at(X4), cc(X6), cg(X7), ga(X9), gc(X10), \\
& & gg(X11), gt(X12), ta(X13), tc(X14), \\
& & $\operatorname{tg}(\mathrm{X} 15), \operatorname{tt}(\mathrm{X} 16)-3 '$ \\
\hline
\end{tabular}

\section{Cluster analysis}

A dendrogram was generated using the $\mathrm{NJ}$ method based on genetic distance. Eighty-seven sweetpea cultivars were grouped into two clusters (Fig. 1). Cluster I consisted of four cultivars, 'Cupani', 'Cupid', 'Painted Lady', and 'Pansy Lavender Flash'. Cluster II consisted of the remaining 83 sweetpea cultivars, and two other Lathyrus species belong to Cluster III as an out-group. It is significant that sweetpea cultivars were grouped into two clusters, I and II, since the genetic relationship
Table 3. AFLP primer pairs and the number of DNA fragments and polymorphic bands used for genetic analysis.

\begin{tabular}{ccc}
\hline \hline Primer & $\begin{array}{c}\text { Number of } \\
\text { DNA } \\
\text { fragments }\end{array}$ & $\begin{array}{c}\text { Number of polymorphic } \\
\text { bands used for genetic } \\
\text { analysis }\end{array}$ \\
\hline MseI-caa/XbaI-cgc & 55 & 6 \\
MseI-cac/XbaI-ccg & 46 & 8 \\
MseI-cac/XbaI-cgc & 39 & 9 \\
MseI-cag/XbaI-cat & 50 & 4 \\
MseI-cag/XbaI-cga & 71 & 13 \\
MseI-cag/XbaI-cgt & 42 & 7 \\
MseI-cat/XbaI-cgc & 52 & 4 \\
MseI-cat/XbaI-cgg & 49 & 16 \\
MseI-cat/XbaI-cgt & 60 & 4 \\
MseI-cat/XbaI-cta & 40 & 5 \\
MseI-cca/XbaI-cac & 64 & 9 \\
MseI-cca/XbaI-cag & 48 & 6 \\
MseI-cca/XbaI-ccg & 54 & 6 \\
MseI-cca/XbaI-cgc & 30 & 4 \\
MseI-ccc/XbaI-ccc & 38 & 6 \\
MseI-ccc/XbaI-cgc & 57 & 6 \\
MseI-ccc/XbaI-cgg & 50 & 9 \\
MseI-ccc/XbaI-cgt & 43 & 7 \\
MseI-ccc/XbaI-ctc & 43 & 5 \\
MseI-ccc/XbaI-ctg & 46 & 13 \\
MseI-ccc/XbaI-ctt & 66 & 12 \\
\hline Total No. & 1043 & 159 \\
No./Primer pair & 49.7 & \\
\hline & & \\
\hline
\end{tabular}




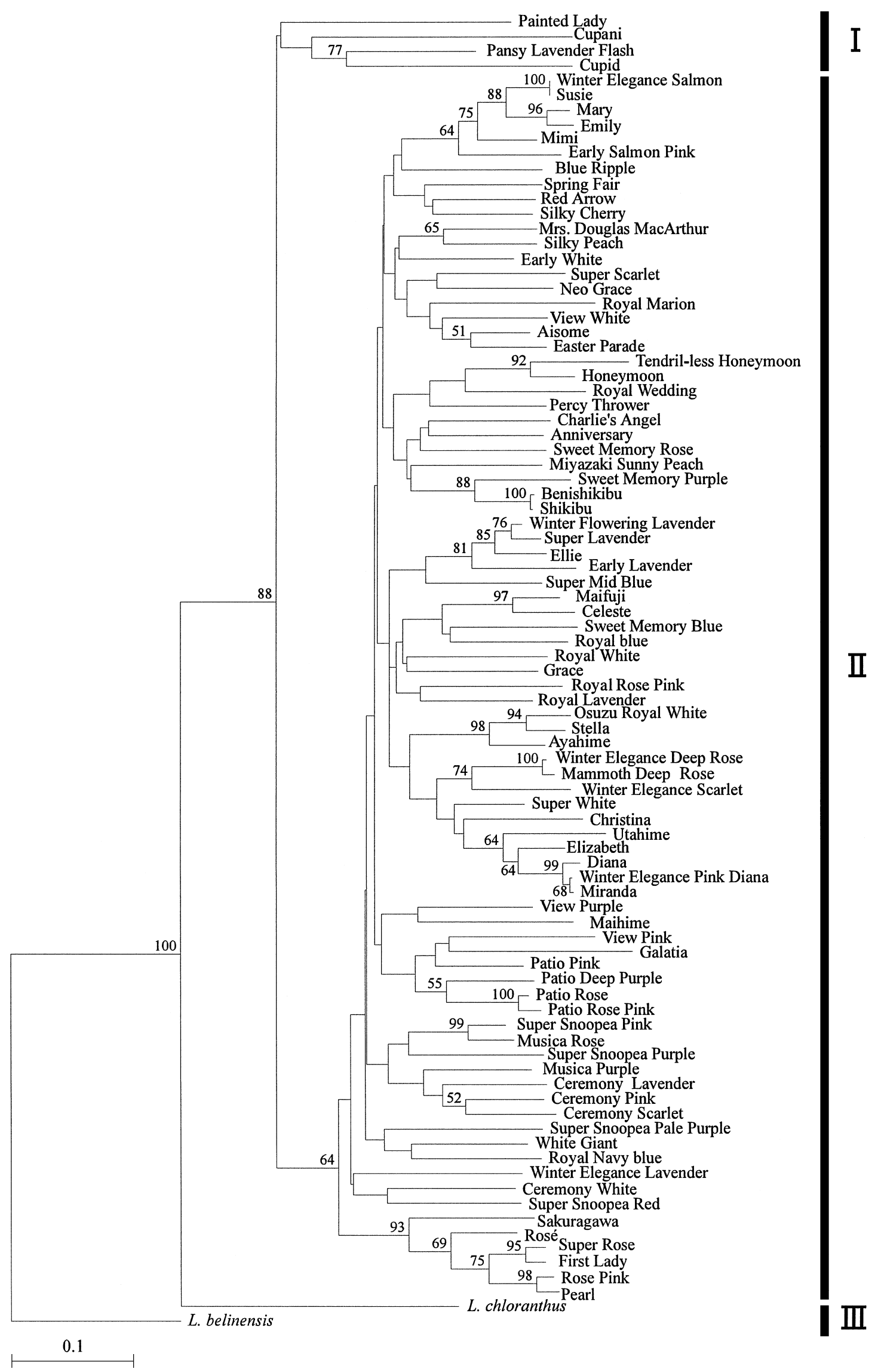

Fig. 1. Dendrogram of 87 sweetpea and 2 related species resulting from neighbor-joining cluster analysis based on simple-matching coefficient calculated from AFLP data. Numbers indicate bootstrap values greater than $50 \%$ for 1000 replicates. 
among sweetpea cultivars was unclear based on morphological and ecological characteristics.

The history of sweetpea, Lathyrus odoratus L., cultivar development is well-documented. Sweetpeas are known to have been introduced from the island of Sicily into cultivation in the United Kingdom in 1699. Since then, many cultivars have been bred in the United Kingdom and the United States (Inoue, 2007; Inoue et al., 2000; Murray and Hammett, 1998; Rice, 2002); however, cultivars were established from spontaneous mutation or natural hybridization until Henry Eckford established the "Grandiflora type" using intraspecific hybridization in 1870s. The "Grandiflora type" has larger and more flowers than cultivars bred before the "Grandiflora type" was established. After the appearance of the "Grandiflora type", many characteristics, such as flower color, flower size, flower form, and flowering habit varied significantly (Inoue, 2007; Inoue et al., 2000; Rice, 2002). Most of the present cultivars were bred after the "Grandiflora type" was established and are classified as "Acacia-leaved", "Cuthbertson", "Dwarf", "Early or Winter-Flowering", "Early Multiflora Giganteas", "Early Spencer", "Galaxy", "Intermediate", "Multiflora", "Royals", "Spencer", and "Unwin type" (Rice, 2002). Most cultivars cultivated in Japan have been selected and fixed from imported market seeds (Inoue, 2007; Nakamura et al., 2008) of "Cuthbertson", "Early Multiflora Giganteas", "Early Spencer", "Early or Winter-Flowering", and "Royals".

Cluster II was characterized by the 83 cultivars that were bred after the "Grandiflora type" was established. This cluster is considered to be the newer type cluster. No distinct subcluster shared common morphological or ecological characteristics. For example, regarding the tendril-less traits, a subcluster consisted of only seven tendril-less cultivars, 'Super Snoopea Pink', 'Musica Rose', 'Super Snoopea Purple', 'Musica Purple', 'Ceremony Lavender', 'Ceremony Pink', and 'Ceremony Scarlet'. However, other tendril-less cultivars, such as 'Tendril-less Honeymoon', 'Super Snoopea Pale Purple', 'Ceremony White', and 'Super Snoopea Red' were grouped into other subclusters. Similarly, neither plant type nor flowering habit formed distinct subclusters. The results suggest that the same mutations occurred among phylogenically different cultivars and lines, and/or the selection and introduction of new characteristics have been repeated through the sweetpea breeding history. Thus, there may be little potential to obtain new useful characteristics within the cultivars of this cluster group except to discover and select new mutations and variations.

Cluster I was considered the cluster of old-type cultivars that were bred before the "Grandiflora type" was established. 'Cupani' and 'Painted Lady' are called "Originals" or "Old fashioned" (Rice, 2002). The reason why cluster I was distinguished from cluster II is considered to be because crossbreeding was not used aggressively between them. 'Cupani', 'Cupid' and 'Painted Lady' were important cultivars in the history of sweetpea improvement. 'Cupani' was introduced from the wild in Sicily in the 1970s (Rice, 2002), and seems to be the same plant that was originally introduced from Sicily to the United Kingdom in 1699. 'Painted Lady' was the first cultivar registered in 1731 and is considered to originate from mutation or natural hybridization from the original sweetpea (Inoue, 2007; Inoue et al., 2000; Murray and Hammett, 1998). 'Cupid', a dwarf cultivar, was a mutant of 'Blanche Ferry' (Beal, 1912b; Rice, 2002) that was developed from 'Painted Lady' (Beal, 1912a, b; Inoue et al., 2000; Rice, 2002). These cultivars were used in the garden or as bedding plants and were not used for cut flowers since the flowers are few and small; however, these cultivars have a simple flower form and fragrance. Inoue et al. (2000) discovered flower color variations of wild individuals in Sicily. Assessment of the variations in these characteristics in old-type cultivars and wild individuals in Sicily would be an important point for breeding new cultivars.

Murray et al. (1992) reported no evidence of change in the chromosome structure and genome size throughout sweetpea development. In this study, sweetpea cultivars were neatly divided into two clusters based on whether they appeared before or after the "Grandiflora type" was established by intraspecific hybridization. We considered that the result reflects the sweetpea development history; that is, sweetpea breeding was promoted by the selection of mutants or natural hybridization in its early history, and then by crossbreeding after the "Grandiflora type" was established.

Cluster III consisted of two Lathyrus species, L. belinensis and L.chloranthus. These species have a yellow flower color that sweetpea does not have, and were reported to form interspecific hybrids with sweetpea (L. odoratus) (Hammet et al., 1994; Khawaja, 1988). Moreover, sweetpea has been reported to form interspecific hybrids with other Lathyrus species, L. cassius and L.hirsutus (Davies, 1957; Inoue, 2007; Murray and Hammett, 1998). It is thus expected that interspecific hybridization is an alternative way to promote the introduction of new useful characteristics to sweetpea.

\section{Literature Cited}

Beal, A. C. 1912a. Sweet pea studies-II. Winter-flowering sweet peas. Bull. Cornell Univ. Coll. Agri. 319: 618-656.

Beal, A. C. 1912b. Sweet pea studies-III. Culture of sweet peas. Bull. Cornell Univ. Coll. Agri. 320: 658-713.

Beal, A. C. 1914. Sweet-pea studies-IV. Classification of garden varieties of the sweet pea. Bull. Cornell Univ. Coll. Agri. 342: 213-360.

Davies, A. J. S. 1957. Successful crossing in the genus Lathyrus through stylar amputation. Nature 180: 612.

Hammett, K. R. W., B. G. Murray, K. R. Markham and I. C. Hallett. 1994. Interspecific hybridization between Lathyrus odoratus and L. belinensis. Int. J. Plant Sci. 155: 763-771. 
Hanada, H. 2003. Classification by polymorphic DNA and development of a genetic marker linked to the tendril trait of sweet pea (Lathyrus odoratus L.). Special Bull. Wakayama. Res. Ctr. Agri. For. Fish. 6: 1-92 (In Japanese).

Hanada, H. and M. Hirai. 2000. Classification of sweet pea (Lathyrus odoratus L.) and everlasting pea (Lathyrus latifolius L.) by random amplified polymorphic DNA (RAPD) analysis. J. Japan. Soc. Hort. Sci. 69: 758-763 (In Japanese with English abstract).

Inoue, T. 2007. Sweet pea wo tsukuri konasu (In Japanese). Nohsangyoson Bunka Kyokai, Tokyo.

Inoue, T., Y. Koike, Y. Miura, H. Higuchi and H. Sasaki. 2000. Growth behavior of sweetpea (Lathyrus odoratus) in Sicily island (the place of origin) and history of differentiation on the flowering lines and varieties. Japan. J. Agric. Educ. 31: 67-74 (In Japanese with English abstract).

Ito, A., T. Inoue, M. Takagi, T. Yamaguchi and K. Yamamoto. 1991. Suitopi. Heisei2nendo shubyotokuseichosahokokusho (In Japanese). Japan seed trade association, Tokyo.

Kawasaki, S. and Y. Murakami. 2000. Genome analysis of Lotus Japonicus. J. Plant Res. 113: 497-506.

Kawasaki, S., Y. Murakami, H. Imaizumi-Anraku, A. Shimizu and I. Mikami. 2003. Construction of high-density map, genome library, and saturation mapping of nodulation genes. p. 183202. In: T. Nagata and S. Tabata (eds.). Biotechnology in agriculture and forestry 52, Brassicas and Legumes. Springer, Berlin.

Khawaja, H. I. T. 1988. A new interspecific Lathyrus hybrid to introduce the yellow flower character into sweet pea. Euphytica 37: 69-75.

Lee, H. J. and T. H. Han. 2006. Identification of parental species of the Alstroemeria cv. 'Jubilee' using AFLP marker technique. Sci. Hortc. 111: 63-67.

Mace, E. S., C. G. Gebhardt and R. N. Lester. 1999. AFLP analysis of genetic relationships in the tribe Dastureae (Solanaceae). Theor. Appl. Genet. 99: 634-641.

Motozu, T. 2006. Sweetpea. p. 256-257. In: Japanese Society for Horticultural Science (ed.). Horticulture in Japan 2006. Shoukabo Publication, Dept. of Publishing of Nakanishi Printing Co., Ltd., Kyoto.

Murray, B. G. and K. R. W. Hammett. 1998. New sweet pea (Lathyrus odoratus) cultivars via interspecific hybridization with wild Lathyrus species. Acta Hort. 454: 115-118.

Murray, B. G., K. R. W. Hammett and L. S. Standring. 1992. Genomic constancy during the development of Lathyrus odoratus cultivars. Heredity 68: 321-327.

Murray, M. and W. F. Thompson. 1980. Rapid isolation of high molecular weight plant DNA. Nucl. Acids Res. 8: 4321-4325.

Nakamura, K., H. Hino, S. Gunji, N. Hattanda, T. Murata, H. Tominaga, K. Fukumoto and R. Akashi. 2008. 'Mimi' Sweet pea for forcing culture. HortScience 43: 2238-2239.

Nakamura, K., K. Fukumoto and R. Akashi. 2010. Genetic variability of morphological and cultural characteristics in sweetpea (Lathyrus odoratus L.). J. Japan. Soc. Hort. Sci. 79. 179-191.

Nakamura, K., K. Shibata, N. Hattanda, T. Murata, S. Gunji, H. Tominaga and H. Takahashi. 2006a. New sweet pea cultivar 'Silky Peach'. Bull. Miyazaki. Agri. Exp. Sta. 41: 131-140 (In Japanese with English abstract).

Nakamura, K., K. Shibata, N. Hattanda, T. Murata, S. Gunji, H. Tominaga and H. Takahashi. 2006b. New sweet pea cultivar 'Silky Cherry'. Bull. Miyazaki. Agri. Exp. Sta. 41: 141-150 (In Japanese with English abstract).

Okawa, K. 1995. Breeding and protection of new varieties of plants. p. 32-49. In: Kakiengeisouron (In Japanese). Yokendo, Tokyo.

Rice, G. 2002. The sweetpea book. B T Bats ford, London.

Saitou, N. and M. Nei. 1987. The neighbor-joining method: A new method for reconstructing phylogenetic trees. Mol. Biol. Evol. 4: 406-425.

Sokal, R. R. and C. D. Michener. 1958. A statistical method for evaluating systematic relationships. Univ. Kansas Sci. Bull. 38: 1409-1438.

Tsuruta, S., R. Akashi, O. Kawamura and H. Numaguchi. 2002. Molecular phylogenetic relationship among fifteen lines of napiergrass (Pennisetum purpureum Schumach.) using RAPD analysis. Grassland Science 48: 37-42 (In Japanese with English abstract).

Van de Peer, Y. and R. De Wachter. 1994. TREECON for Windows: a software package for the construction and drawing of evolutionary trees for the Microsoft Windows environment. Comput. Applic. Biosci. 10: 569-570.

Vos, P., R. Hogers, M. Bleeker, M. Reijans, T. Lee, M. Hornes, A. Frijters, J. Pot, J. Peleman, M. Kuiper and M. Zabeau. 1995. AFLP: a new technique for DNA fingerprinting. Nucl. Acids Res. 23: 4407-4414.

Warwick, S. I., R. K. Gugel, C. Gómez-Campo and T. James. 2007. Genetic variation in Eruca vesicaria (L.) Cav. Plant Genetic Resources 5: 142-153. 